\title{
Plant species identification using shortest paths in graphs applied to color images of palisade parenchyma
}

\author{
Jarbas Joaci de Mesquita Sá Junior ${ }^{\mathrm{a}}$, Paulo César Cortez ${ }^{\mathrm{b}}$, Rosana Marta Kolb ${ }^{\mathrm{c}}$, \\ André Ricardo Backes ${ }^{\mathrm{d},}$, \\ a Curso de Engenharia de Computação, Universidade Federal do Ceará - Campus de Sobral, Rua Estanislau Frota, S/N, Centro, Sobral CEP: 62010-560, Cearã, Brazil \\ b Departamento de Engenharia de Teleinformática, Universidade Federal do Ceará, Campus do Pici, S/N, Bloco 725, Caixa Postal 6007, Fortaleza CEP: 60.455-970, \\ Ceará, Brazil \\ c Departamento de Ciências Biológicas, Faculdade de Ciências e Letras, Universidade Estadual Paulista, Av. Dom Antônio, 2100, Assis CEP: 19806-900, SP, Brazil \\ d Faculdade de Computação, Universidade Federal de Uberlândia, Av. João Naves de Ávila, 2121, Uberlândia CEP: 38408-100, MG, Brazil
}

A R T I C L E I N F O

\section{Keywords:}

Plant identification

Color texture

Palisade parenchyma

Shortest paths

Graphs

\begin{abstract}
A B S T R A C T
Aiming to identify plants, we propose to evaluate the color texture of the palisade parenchyma, from microscopic images of leaf cross-sections, using a graph based approach. Our texture analysis approach models the image texture as a graph and uses measurements computed from the shortest paths between specific vertices to provide a feasible texture signature. For a more consistent evaluation, we compared our approach to different methods for color texture analysis in a texture classification experiment. The results obtained indicate that our approach is the most suitable for this histological analysis as it surpassed all the other texture approaches using Linear Discriminant Analysis, and obtained the second best accuracy using 1-Nearest Neighbor. These results also corroborate the feasibility of using both histological (as the palisade parenchyma) and computer analysis for identification and delimitation of plant taxa.
\end{abstract}

\section{Introduction}

Texture is one of the most important features in image analysis field, thus promoting intensive research of approaches that extract more discriminative image descriptors. Although there is a lack of formal definition, texture is usually defined as visual patterns composed of subpatterns (texture primitives) with specific size, orientation, brightness, color, etc. This definition, however, is not suitable for natural textures (e.g., clouds and smoke) as they present a random and persistent stochastic pattern, thus resulting in a cloud like texture appearance (Kaplan, 1999).

Another important attribute of images is color and there is extensive discussion whether color and texture information should be considered jointly or separately (Drimbarean and Whelan, 2001; Bianconi et al., 2011). The approach in Backes et al. (2012) used fractal descriptors to investigate the complexity in R, G and B color channels to characterize a texture sample. This approach is also used in the combinations of RGB channels, taking into consideration the correlations among them. Drimbarean and Whelan (2001) aimed to determine the contribution of color information to the overall classification performance. This was performed by evaluating the contribution of color and texture features separately and collectively, as well as the effect of using different color spaces. In Bianconi et al. (2011) the authors performed a comprehensive comparison, from both a theoretical and experimental standpoint, of the use of color and texture information jointly and separately. As it turns out, there is no standard procedure for processing color textures, and, basically, the methods have been grouped into three categories: parallel (color and texture are processed separately); sequential (colors are indexed in order to construct images that can be processed as grayscale textures); and integrative (color and texture are processed jointly) (Palm, 2004).

Traditional identification of plants is a complex task, consuming time and depending on knowledge of few specialists. In this regard, automated plant identification has become the subject of intensive research in computer vision. Moreover, computer vision systems can quantify characteristics not considered in traditional plant taxonomy, such as color, texture and shape, thus providing novel tools to this knowledge field.

Among the plant organs used for taxonomic purposes, leaf has proven to be a rich information source. For instance, Casanova et al. (2009) applied Gabor filters in grayscale textures from leaf surfaces. Plotze et al. (2005) analyzed the leaf shape and venation from 10

\footnotetext{
* Corresponding author.

E-mail addresses: jarbas_joaci@yahoo.com.br (J.J.d.M. Sá Junior), cortez@lesc.ufc.br (P.C. Cortez), rosanakolb@hotmail.com (R.M. Kolb), backes@ufu.br (A.R. Backes).
} 
species of the genus Passiflora by using fractal dimension. The papers of Sá Junior et al. $(2011,2013)$ explored the internal structure of leaves (palisade parenchyma, spongy parenchyma, adaxial face of epidermis and its cuticle) from microscopic images of their cross-sections.

The present work proposes to apply a recent integrative color texture analysis method based on shortest paths in graphs to images of palisade parenchyma cells in order to identify plants. This approach was proposed for two reasons. First, this texture analysis algorithm, proposed in the paper of Sá Junior et al. (2014), has presented excellent success rates in three well-known color texture benchmarks, proving to be able to provide discriminative signatures. Second, parenchyma palisade has proven to be a very informative plant tissue according to the papers of Sá Junior et al. (2011, 2013).

The paper is organized as follows: Section 2 explains how to convert a color texture image into an undirected graph. In Section 3, we show the procedure of extracting signatures from shortest paths in graphs. Section 4 details the palisade parenchyma dataset, explains the experimental procedure, and discusses the results obtained. Finally, Section 5 presents some remarks on this study.

\section{Color texture modeled as a graph}

To model a texture as an undirected graph $G(V, E)$, each pixel $I(x, y)$ is interpreted as a vertex $v \in V$. An undirected edge $e \in E$ connects two vertices if the Chebyshev distance between them is smaller than or equal to 1 , that is,

$E=\left\{e=\left(v, v^{\prime}\right) \in V \times V \mid \max \left(\left|x-x^{\prime}\right|,\left|y-y^{\prime}\right|\right) \leq 1\right\}$,

where $x$ and $y$ are the Cartesian coordinates of the pixel $I(x, y)$. Each edge has a weight $w(e)$ determined according to the following equation:

$w(e)=\left|I(x, y)-I\left(x^{\prime}, y^{\prime}\right)\right|+\left(I(x, y)+I\left(x^{\prime}, y^{\prime}\right)\right) / 2$,

where $I(x, y)=g, g \in\{0, \ldots, 255\}$ is the intensity of the pixel $I(x, y)$. Notice that the weight of an edge consists of two parts. The first part emphasizes the absolute difference between pixels, so that it privileges vertices associated to similar pixels and avoids abrupt changes in the image. The second part emphasizes the search for lower levels of intensity, a characteristic that may be related to change in the illumination or borders in the image.

The $R G B$ color texture was converted into a graph in two manners. In the first approach, each color channel is an independent graph. For each color channel $I_{C}(x, y), C \in\{R, G, B\}$, a graph is built according to Eq. (1). In the second approach, the three color channels are considered altogether and only one graph is constructed. In this unique graph, two vertices $v$ and $v^{\prime}$ representing $I_{a}(x, y)$ and $I_{b}\left(x^{\prime}, y^{\prime}\right)$, respectively, where $a, b \in\{R, G, B\}$, are connected by an undirected edge $e \in E$ if the Chebyshev distance is also shorter than or equal to 1 , but subject to $(a=b) \vee(a=G \wedge b \in\{R, B\})$. Notice that we use channel $G$ as a bridge connecting the other two channels. Thus, we force image exploration to a multi-channel level, so that the shortest paths are able to hold information about the transitions between channels. In both approaches, the weight edge $w(e)$ is determined according to Eq. (2). More details can be found in the paper of Sá Junior et al. (2014).

\section{Shortest path signatures for color textures}

To extract a color signature from a graph, we propose to use shortest paths computed by Dijkstra's algorithm (Dijkstra, 1959). For this purpose, each color channel has four sets of starting and respective ending vertices, called horizontal points (path $p_{0^{\circ}}$ ), vertical points (path $p_{90^{\circ}}$ ) and diagonal points (paths $p_{45^{\circ}}$ and $p_{135^{\circ}}$ ), as shown in Fig. 1 .

Even though both strategies for graph modeling have the same sets of starting and ending vertices, their shortest paths present different behavior. In the first approach "one graph per color channel", a shortest path always belongs to a same color channel. On the other hand, in the approach "one graph for the whole image", a shortest path always starts and ends in the same color channel, but can explore other channels along the path. Thus, it is possible to extract textural information from each color channel and explore the interactions among the image colors.

In order to obtain a signature that contains both local and global characteristics, two groups of feature vectors are proposed. In the first group, the image is covered by a grid of disjoint windows $l \times l$ (disjoint windows are used to decrease the computational cost of the method). For each window and color channel $C \in\{R, G, B\}$, we compute the four shortest paths $\left(p_{0^{\circ}}, p_{45^{\circ}}, p_{90^{\circ}}\right.$ and $\left.p_{135^{\circ}}\right)$. Next, we compute average $\mu_{d^{\circ}}$ and standard deviation $\sigma_{d^{\circ}}$ in a same direction $d \in\{0,45,90,135\}$.

Thus, we propose the following feature vectors:

$\vec{\alpha}_{l}^{C}=\left[\mu_{0}{ }^{\circ}, \sigma_{0}{ }^{\circ}, \mu_{45^{\circ}}, \sigma_{45^{\circ}}, \mu_{90^{\circ}}, \sigma_{90^{\circ}}, \mu_{135^{\circ}}, \sigma_{135^{\circ}}\right]$,

and

$\vec{\beta}_{l}^{C}=\left[\mu_{0}{ }^{\circ}, \sigma_{0}{ }^{\circ}, \mu_{45^{\circ}}, \sigma_{45^{\circ}}, \mu_{90^{\circ}}, \sigma_{90^{\circ}}, \mu_{135^{\circ}}, \sigma_{135^{\circ}}\right]$,

where $\vec{\alpha}_{l}$ and $\overrightarrow{\beta_{l}}$ represent the approaches "one graph per color channel" and "one graph for the whole image", respectively. Notice that both $\vec{\alpha}_{l}$ and $\overrightarrow{\beta_{l}}$ contains 8 descriptors (average and standard deviation for each of the 4 directions considered).

By considering a window size $l$ and the three color channels, the following feature vectors can be obtained. Feature vectors $\vec{\theta}_{l}$ and $\vec{\psi}_{l}$ contain 24 descriptors ( 8 descriptors for each RGB color channel), while $\vec{\omega}_{l}$ represents their concatenation, totalising 48 descriptors:

$\vec{\theta}_{l}=\left[\vec{\alpha}_{l}^{R}, \vec{\alpha}_{l}^{G}, \vec{\alpha}_{l}^{B}\right]$,

$\vec{\psi}_{l}=\left[\vec{\beta}_{l}^{R}, \vec{\beta}_{l}^{G}, \vec{\beta}_{l}^{B}\right]$,

and

$\vec{\omega}_{l}=\left[\vec{\theta}_{l}, \vec{\psi}_{l}\right]$

In the second group of feature vectors, which aims to achieve a multi-scale approach for color texture characterization, it is important to consider multiple window sizes $l$. This can be easily achieved by concatenating the previous feature vectors, as described below. In this case, feature vectors $\vec{\Theta}$ and $\vec{\Psi}$ contain 24 descriptors for each window size $l_{i}, i=1, \ldots, n$, while their concatenation, $\vec{\Omega}$, contains 48 descriptors for each window size:

$\vec{\Theta}_{l_{1}, l_{2}, \ldots, l_{n}}=\left[\vec{\theta}_{l_{1}}, \vec{\theta}_{l_{2}}, \ldots, \vec{\theta}_{l_{n}}\right]$,

$\vec{\Psi}_{l_{1}, l_{2}, \ldots, l_{n}}=\left[\vec{\psi}_{l_{1}}, \vec{\psi}_{l_{2}}, \ldots, \vec{\psi}_{l_{n}}\right]$,

$\vec{\Omega}_{l_{1}, l_{2}, \ldots, l_{n}}=\left[\vec{\Psi}_{l_{1}, l_{2}, \ldots, l_{n}}, \vec{\Theta}_{l_{1}, l_{2}, \ldots, l_{n}}\right]$.

\section{Experiment and results}

The database used in this work is composed of 320 images $60 \times 60$ pixel size of palisade parenchyma tissue divided into 8 classes. Each class contains 40 samples obtained from 10 randomly chosen individuals of each species. The classes represent the plant species Byrsonima intermedia, Miconia albicans, Tibouchina stenocarpa, Vochysia tucanorum, Xylopia aromatica, Moquiniastrum polymorphum (= Gochnatia polymorpha), Miconia chamissois and Jacaranda caroba. For obtaining the images, all leaf samples were fixed in formalin-acetic acid-alcohol 70\% (FAA 70), dehydrated in a graded ethanol series, infiltrated and embedded in paraffin and cut into $8 \mu \mathrm{m}$ sections. In the sequence, the cross-sections were stained with astra blue-basic fuchsine and permanently mounted in entellan. For image acquisition, we used a trinocular microscope Leica, model DM-1000, coupled with a video camera Leica, DFC-280, using $20 \times$ objective lenses. Fig. 2 shows one 

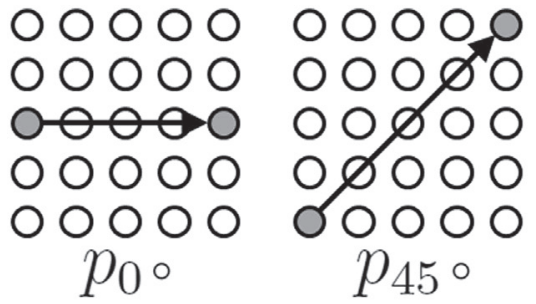

Fig. 1. The four sets of vertices used to compute the shortest paths.

Source: Modified from Sá Junior et al. (2014).

sample of each class. The signatures provided by our texture analysis method were classified using 1-Nearest Neighbor (1-NN) and Linear Discriminant Analysis (LDA) (Fukunaga, 1990) in a leave-one-out cross validation strategy.

In our proposed texture analysis method, computing the shortest paths can be a time consuming task if the number of vertices and edges in a graph is large. Thus, to extract the shortest paths from the image graphs, we divided each image sample $(60 \times 60$ pixels $)$ into disjoint windows of $l \times l$ pixel size. Since the $l$ value affects the calculus of the average and standard deviation used in the proposed signatures, we performed several tests with different window sizes $l$, as shown in Table 1. Results show that the performance of the method tends to reduce as the $l$ values increase. This can be explained by two reasons. First, a larger $l$ value results in a smaller number of windows used to subdivide the texture. Second, each window may contain regions of the texture that present different aspects. Thus, because the proposed descriptors compress different aspects of the texture into a single pair of values (average and standard deviation), a bad sampling of different regions of the texture may cause a loss of discrimination ability of the method.

This experiment also showed that the approach "one graph per channel" is more efficient than the approach "one graph for the whole image" for the characterization of the palisade parenchyma database used in this study (except for the window size $l=4$ ). Also, it demonstrated that the synergism of the vectors $\vec{\theta}_{l}$ and $\vec{\psi}_{l}$ not always increases the capacity of discriminating the samples.

Different window sizes affect how the texture is sampled by the method. This indicates a multi-resolution behavior of the method. Thus, it may be interesting to evaluate the performance of the method as we combine the signatures computed for multiple $l$ values, as shown in Table 2. Unlike the experiments presented in the paper of Sá Junior

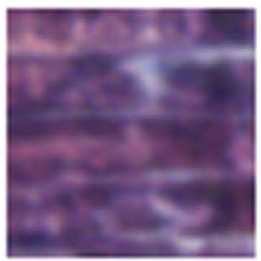

(a)

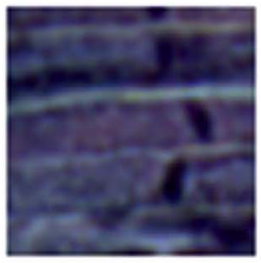

(e)

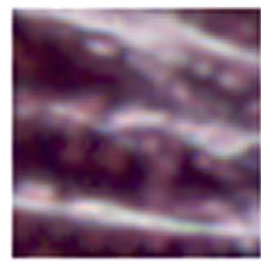

(b)

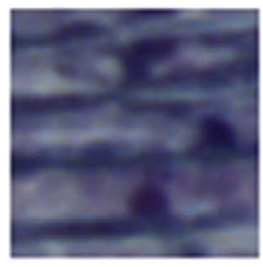

(f)
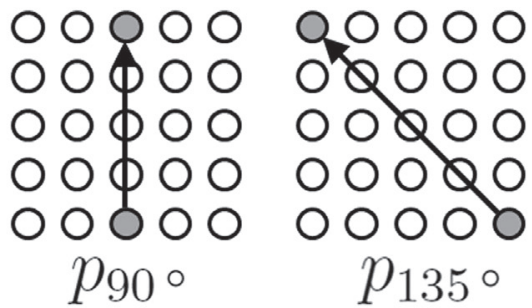

Table 1

Success rate (\%) of the shortest paths in graphs method applied to the palisade parenchyma database for different window sizes $l$ using 1 -NN.

\begin{tabular}{|c|c|c|c|c|c|c|c|c|}
\hline \multirow[b]{2}{*}{$l$} & \multicolumn{8}{|c|}{ Success rate $(\%)$} \\
\hline & 4 & 5 & 6 & 10 & 12 & 15 & 20 & 30 \\
\hline $\overrightarrow{\theta_{l}}$ & 81.25 & 83.13 & 82.81 & 78.44 & 79.69 & 79.38 & 78.13 & 76.88 \\
\hline $\overrightarrow{\psi_{l}}$ & 84.06 & 81.88 & 76.56 & 64.69 & 59.69 & 51.88 & 45.63 & 39.06 \\
\hline$\vec{\omega}_{l}$ & 86.56 & 82.81 & 85.63 & 76.88 & 76.88 & 80.63 & 70.63 & 65.31 \\
\hline
\end{tabular}

Table 2

Success rate (\%) of the shortest paths in graphs method applied to the palisade parenchyma database for different sets of window sizes $l$ using 1-NN.

\begin{tabular}{llll}
\hline \multicolumn{4}{l}{ Success rate (\%) } \\
\hline Set of window sizes $\left\{l_{1}, l_{2}, \ldots, l_{n}\right\}$ & $\vec{\Theta}_{l_{1}, l_{2}, \ldots, l_{n}}$ & $\vec{\Psi}_{l_{1}, l_{2}, \ldots, l_{n}}$ & $\vec{\Omega}_{l_{1}, l_{2}, \ldots, l_{n}}$ \\
$\{4,5\}$ & 83.13 & 84.69 & 85.63 \\
$\{4,5,6\}$ & 82.50 & 83.13 & 85.94 \\
$\{4,5,6,10\}$ & 80.94 & 83.13 & 86.25 \\
$\{4,5,6,10,12\}$ & 82.19 & 80.31 & 84.69 \\
$\{4,5,6,10,12,15\}$ & 81.88 & 76.88 & 84.69 \\
$\{4,5,6,10,12,15,20\}$ & 81.88 & 76.56 & - \\
$\{4,5,6,10,12,15,20,30\}$ & 82.81 & 77.19 & - \\
\hline
\end{tabular}

et al. (2014), the use of sets of values $l$ tends to decrease the success rate. Thus, none of the success rates surpassed the result obtained by the feature vector $\vec{\omega}_{4}$, which is $86,56 \%$. One possible explanation for such results is the size of the original samples $(60 \times 60$ pixel size). We are working with small samples of a very similar plant tissue. Thus, even though the signatures computed for different $l$ values may be different, they do not hold enough difference between each other to

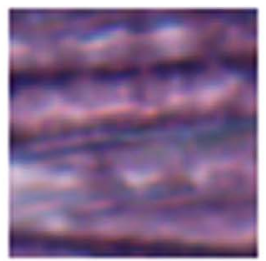

(c)

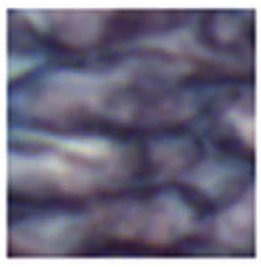

(g)

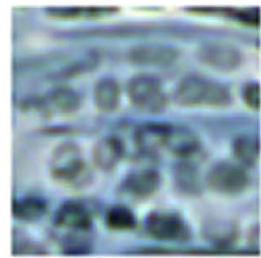

(d)

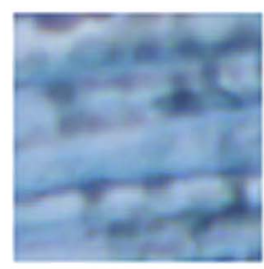

(h)

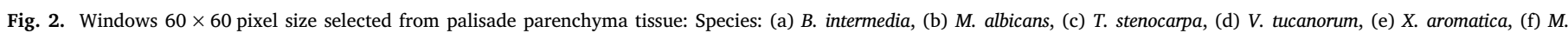
polymorphum, (g) M. chamissois and (h) J. caroba. 
Table 3

Comparison results of different methods applied to the palisade parenchyma database.

\begin{tabular}{llll}
\hline Methods & No. of descriptors & \multicolumn{2}{l}{ Success rate (\%) } \\
\hline & & LDA & $1-\mathrm{NN}$ \\
Shortest paths in graphs $R G B$ & 48 & 91.56 & 86.56 \\
Gabor EEE & 192 & 87.81 & 92.50 \\
HRF & - & 33.12 & 33.12 \\
Multilayer CCR & 640 & 91.25 & 79.38 \\
MSD & 72 & 82.19 & 73.13 \\
LBP + Haralick & 10 & 60.62 & 67.50 \\
LBP & 54 & 72.19 & 57.50 \\
CLBP & 648 & 76.56 & 76.56 \\
\hline
\end{tabular}

improve the classification when combined.

To improve the evaluation of our method, we also proposed a comparison with traditional texture analysis methods found in literature. For this comparison, we included five color texture analysis methods: Gabor EEE (Hoang and Geusebroek, 2002; Hoang et al., 2005), HRF (Paschos and Petrou, 2003) (it has its own classification scheme), Multilayer CCR (Bianconi et al., 2009), MSD (Liu et al., 2011) and "LBP + Haralick" (Porebski et al., 2008). We also included, for comparison and baseline, two grey-scale texture descriptors: Local Binary Patterns (LBP) (Ojala et al., 2002) and Completed Local Binary Patterns (CLBP) (Guo et al., 2010) (it has its own classification scheme).

Taking into account the previous results, we adopted the signature $\vec{\omega}_{4}$, which provides the highest success rate in Table 1 , to compare our signature using 1-NN and LDA. As we can notice in Table 3, the performance of our approach surpassed all the results of the compared methods using LDA and obtained the second best accuracy using 1-NN. Also, it is worth mentioning the small number of used descriptors (48), which is only greater than the number of descriptors of the "LBP + Haralick" method. For instance, "Multilayer CCR" method uses 640 descriptors to obtain the closest success rate to ours and Gabor EEE uses 192 descriptors to overcome our method, thus making our result statistically significant. Results also show that color is an important information to be considered as grey-scale baseline methods (LBP (Ojala et al., 2002) and CLBP (Guo et al., 2010)) perform poorly in this problem.

\section{Conclusion}

Plant images in general, and in particular images of palisade parenchyma tissue, represent a difficult classification problem for image analysis methods. This is mostly due to the high similarity among samples from different species and irregularities within a same species. In order to address this problem, this paper presents a recent and powerful approach to extract features from a texture pattern. We compared our approach to different methods for color texture analysis. If we considered the highest number of correctly classified samples, together with the fewest descriptors needed, the results of our approach surpassed those of the other methods compared. In addition, the results obtained demonstrated that the histological analysis of the palisade parenchyma is suitable for plant identification.

\section{Acknowledgments}

André R. Backes gratefully acknowledges the financial support of CNPq (National Council for Scientific and Technological Development, Brazil) (Grant \#302416/2015-3), FAPEMIG (Foundation to the Support of Research in Minas Gerais) (Grant \#APQ-03437-15) and PROPP-UFU.

\section{References}

Backes, A.R.A.R., Casanova, D.D., Bruno, O.M.O.M., 2012. Color texture analysis based on fractal descriptors. Pattern Recogn. 45 (5), 1984-1992.

Bianconi, F.F., Fernández, A.A., González, E.E., Caride, D.D., Calvino, A.A., 2009. Rotation-invariant colour texture classification through multilayer CCR. Pattern Recogn. Lett. 30 (8), 765-773.

Bianconi, F.F., Harvey, R.R., Southam, P.P., Fernández, A.A., 2011. Theoretical and experimental comparison of different approaches for color texture classification. J. Electron. Imaging 20 (4), 043006.

Casanova, D.D., Sá Junior, J.J.M.J.J.M., Bruno, O.M.O.M., 2009. Plant leaf identification using Gabor wavelets. Int. J. Imaging Syst. Technol. 19 (1), 236-243.

Dijkstra, E.W.E.W., 1959. A note on two problems in connexion with graphs. Numer. Math. 1, 269-271. http://gdzdoc.sub.uni-goettingen.de/sub/digbib/ loader?did=D196313.

Drimbarean, A.A., Whelan, P.F.P.F., 2001. Experiments in colour texture analysis. Pattern Recogn. Lett. 22 (10), 1161-1167.

Fukunaga, K.K., 1990. Introduction to Statistical Pattern Recognition, 2nd edition. Academic Press, San Diego, CA, USA.

Guo, Z.Z., Zhang, L.L., Zhang, D.D., 2010. A completed modeling of local binary pattern operator for texture classification. IEEE Trans. Image Process. 19 (6), 1657-1663.

Hoang, M.A.M.A., Geusebroek, J.M.J.M., 2002. Measurement of color texture. In: Workshop on Texture Analysis in Machine Vision, pp. 73-76.

Hoang, M.A.M.A., Geusebroek, J.-M.J.-M., Smeulders, A.W.M.A.W.M., 2005. Color texture measurement and segmentation. Signal Process. 85 (2), 265-275.

Kaplan, L.M.L.M., 1999. Extended fractal analysis for texture classification and segmentation. IEEE Trans. Image Process. 8 (11), 1572-1585.

Liu, G.-H.G.-H., Li, Z.Z., Zhang, L.L., Xu, Y.Y., 2011. Image retrieval based on microstructure descriptor. Pattern Recogn. 44 (9), 2123-2133.

Ojala, T.T., Pietikainen, M.M., Maenpaa, T.T., 2002. Multiresolution gray-scale and rotation invariant texture classification with local binary patterns. IEEE Trans. Pattern Anal. Mach. Intell. 24 (7), 971-987.

Palm, C.C., 2004. Color texture classification by integrative co-occurrence matrices. Pattern Recogn. 37 (5), 965-976.

Paschos, G.G., Petrou, M.M., 2003. Histogram ratio features for color texture classification. Pattern Recogn. Lett. 24 (1-3), 309-314.

Plotze, R.O.R.O., Pádua, J.G.J.G., Falvo, M.M., Bernacci, L.C.L.C., Oliveira, G.C.X.G.C.X., Vieira, M.L.C.M.L.C., Bruno, O.M.O.M., 2005. Leaf shape analysis using the multiscale Minkowski fractal dimension, a new morphometric method: a study with Passiflora (Passifloraceae). Can. J. Bot. - Rev. Can. Bot. 83 (3), 287-301.

Porebski, A.A., Vandenbroucke, N.N., Macaire, L.L., 2008. Haralick feature extraction from LBP images for color texture classification. In: Imag. Process. Theory, Tools Appl. pp. 1-8.

Sá Junior, J.J.M.J.J.M., Backes, A.R.A.R., Cortez, P.C.P.C., 2014. Color texture classification using shortest paths in graphs. IEEE Trans. Image Process. 23 (9), 3751-3761.

Sá Junior, J.J.M.J.J.M., Backes, A.R.A.R., Rossatto, D.R.D.R., Kolb, R.M.R.M., Bruno, O.M.O.M., 2011. Measuring and analyzing color and texture information in anatomical leaf cross sections: an approach using computer vision to aid plant species identification. Botany 89 (7), 467-479.

Sá Junior, J.J.M.J.J.M., Rossatto, D.R.D.R., Kolb, R.M.R.M., Bruno, O.M.O.M., 2013. A computer vision approach to quantify leaf anatomical plasticity: a case study on Gochnatia polymorpha (Less.) Cabrera. Eco. Inform. 15, 34-43. 